\title{
Universal zero solutions of linear partial differential operators
}

\author{
by \\ Thomas Kalmes (Trier) and Markus Niess (Eichstätt)
}

\begin{abstract}
A generalized approach to several universality results is given by replacing holomorphic or harmonic functions by zero solutions of arbitrary linear partial differential operators. Instead of the approximation theorems of Runge and others, we use an approximation theorem of Hörmander.
\end{abstract}

1. Introduction. The first universality result in complex analysis is the famous theorem of G. D. Birkhoff [3], which (slightly modified) reads as follows:

THEOREM 1.1 (Birkhoff (1929)). There exists an entire function u such that for every entire function $f$, every compact set $K \subset \mathbb{C}$ and for every $\varepsilon>0$, there is a $p \in \mathbb{N}$ satisfying

$$
|u(z+p)-f(z)|<\varepsilon \quad \text { for all } z \in K .
$$

We then say that $u$ has universal translates. Analogues were obtained by Seidel and Walsh [14] for non-Euclidean translates in the unit disk $\mathbb{D}$ in 1941 , and for dilations $u(p \cdot z)$, with $p \in \mathbb{C}^{*}:=\mathbb{C} \backslash\{0\}$, instead of translates by Zappa [16] in 1989. Using linear transformations $u(a \cdot z+b)$ Luh [1] generalized the concept of universality to simply connected domains in 1979, and to arbitrary domains later on with his colleagues. The residuality of the corresponding sets of universal functions was discovered by Duyos-Ruiz [5] in 1984.

A further generalization is due to Bernal and Montes [2] (1995), who considered composition operators induced by a sequence $\left(f_{n}\right)$ of conformal automorphisms on a general open $\Omega \subset \mathbb{C}$. Such a sequence is called run-away if for every compact subset $K$ of $\Omega$ there is some $n \in \mathbb{N}$ with $K \cap f_{n}(K)=\emptyset$. In these terms, they stated the following result.

2010 Mathematics Subject Classification: Primary 47A16, 46E10, 46F05; Secondary 35D99. Key words and phrases: universality, hypercyclic sequence, dense subspace of universal elements, composition operators, kernels of linear differential operators. 
Theorem 1.2 (Bernal and Montes (1995)). Let $\Omega \subset \mathbb{C}$ be an open set that is not conformally equivalent to $\mathbb{C}^{*}$, and let $\left(f_{n}\right)$ be a sequence of automorphisms of $\Omega$. Then there exists a function u holomorphic on $\Omega$ for which the set $\left\{u \circ f_{n}: n \in \mathbb{N}\right\}$ is dense in $H(\Omega)$, the space of all holomorphic functions on $\Omega$, if and only if $\left(f_{n}\right)$ is a run-away sequence. In that case the set of such functions $u$ is residual in $H(\Omega)$.

The first universality result in harmonic analysis is due to Dzagnidze [6] (1969) and is the harmonic analogue of Birkhoff's Theorem.

Our aim is to give a generalized approach and proof of all these universality results. That is, instead of considering the special polynomials

$$
P_{1}: \mathbb{R}^{2} \rightarrow \mathbb{C}, \xi \mapsto \frac{1}{2}\left(\xi_{1}+i \xi_{2}\right), \quad \text { and } \quad P_{2}: \mathbb{R}^{N} \rightarrow \mathbb{C}, \xi \mapsto \sum_{j=1}^{N} \xi_{j}^{2},
$$

which give $P_{1}(\partial) f=\bar{\partial} f$, i.e. the Cauchy-Riemann operator, and $P_{2}(\partial) f=$ $\Delta f$, the Laplacian, respectively, we consider arbitrary differential operators $P(\partial)$ and their kernels, where $P$ is a non-constant polynomial on $\mathbb{R}^{N}$ with complex coefficients. We are interested in properties of sequences $\left(f_{m}\right)_{m \in \mathbb{N}}$ of diffeomorphisms of $\Omega$ such that there is an element $u$ of the kernel of $P(\partial)$ such that $\left\{u \circ f_{m} ; m \in \mathbb{N}\right\}$ is dense in the kernel.

As domain of definition of the operator $P(\partial)$ we choose the Fréchet spaces $\bigcap_{j=1}^{\infty} B_{p_{j}, k_{j}}^{\text {loc }}(\Omega)$ introduced by Hörmander [10] (see Section 2). As a special case, they include the space $C^{\infty}(\Omega)$ equipped with its standard Fréchet space topology, i.e. local uniform convergence of all partial derivatives, which we denote as usual by $\mathscr{E}(\Omega)$. Since the kernels of $P_{1}(\partial)$ and $P_{2}(\partial)$ considered as operators on $\mathscr{E}(\Omega)$ are the space $H(\Omega)$ of holomorphic functions on $\Omega \subset \mathbb{R}^{2} \cong \mathbb{C}$ and the space $h(\Omega)$ of harmonic functions on $\Omega \subset \mathbb{R}^{N}$, respectively, holomorphic as well as harmonic universal functions are covered by this framework. (Note that by a standard application of the Open Mapping Theorem for Fréchet spaces, the topologies inherited from $\mathscr{E}(\Omega)$ are indeed the usual Fréchet space topologies on $H(\Omega)$ and $h(\Omega)$, respectively!)

The price we have to pay for this generality is that we lose special structures of the function spaces. Instead of the approximation theorems of Runge and others, we use a general approximation theorem due to Hörmander (cf. Theorem 4.2 which forces us to impose stronger geometrical conditions on $\Omega$, namely we assume the components of $\Omega$ to be convex.

A similar approach in the case of translations has been taken by CalderónMoreno and Müller [4]. They use the famous Lax-Malgrange theorem which guarantees less losses in the structure of the open sets $\Omega$, but one is restricted to elliptic partial differential operators.

Finally, we also want to mention two recent and different directions that are related to the above mentioned results. Gauthier and Pouryayevali [7] 
obtained universal subharmonic functions on $\mathbb{R}^{N}$ and universal plurisubharmonic functions on $\mathbb{C}^{N}$. They understand the universality in the sense of Birkhoff. Grosse-Erdmann and Mortini [9] worked on an analogue of Theorem 1.2 but for sequences $\left(f_{n}\right)$ of eventually injective or arbitrary holomorphic self-maps of $\Omega$.

The paper is organized as follows. In Section 2 we recall some facts about the Fréchet spaces $\bigcap_{j=1}^{\infty} B_{p_{j}, k_{j}}^{\text {loc }}(\Omega)$. In Section 3 we consider the kernel of $P(\partial)$ as a subspace of $\bigcap_{j=1}^{\infty} B_{p_{j}, k_{j}}^{\text {loc }}(\Omega)$ and introduce composition operators $u \mapsto u \circ f$ on these kernels. It turns out that in general $f$ can only be chosen from a very small class of diffeomorphisms in order to ensure that the composition operator is well-defined. Section 4 contains a sufficient condition on the sequence $\left(f_{m}\right)_{m \in \mathbb{N}}$ of diffeomorphisms ensuring the existence of universal zero solutions of $P(\partial)$ as well as a result analogous to Theorem 1.2 . Finally, Section 5 deals with dense subspaces of universal zero solutions.

Throughout the paper, we use the following notations. The interior of a subset $M$ of $\mathbb{R}^{N}$ is denoted by $M^{\circ}$. The Fourier transform of a tempered distribution $u$ is denoted by $\hat{u}$ or $\mathcal{F}(u)$ (where for $u \in L^{1}\left(\mathbb{R}^{N}\right)$ we set $\hat{u}(\xi)=$ $\left.\int e^{-i\langle x, \xi\rangle} u(x) d x\right)$. For a topological vector space $E$ its topological dual $E^{\prime}$ is always equipped with the weak*-topology, and by a diffeomorphism we always mean a $C^{\infty}$-diffeomorphism. As usual, we denote by $\mathscr{S}^{\prime}$ the space of tempered distributions on $\mathbb{R}^{N} ; \mathscr{D}(\Omega)$ is the space of compactly supported $C^{\infty}$-functions on $\Omega$ equipped with its usual inductive limit topology, and $\mathscr{D}^{\prime}(\Omega)$ its dual, i.e. the space of distributions on $\Omega$; and for an arbitrary subset $A$ of $\mathbb{R}^{N}, \mathscr{E}^{\prime}(A)$ denotes the space of distributions on $\mathbb{R}^{N}$ having compact support in $A$. Besides that, we use the standard notation from functional analysis (see e.g. [12, 13]).

2. The Fréchet space $\bigcap_{j=1}^{\infty} B_{p_{j}, k_{j}}^{\text {loc }}(\Omega)$. In this section we recall some facts about the spaces $\bigcap_{j=1}^{\infty} B_{p_{j}, k_{j}}^{\text {loc }}(\Omega)$ for $\Omega \subset \mathbb{R}^{N}$ open, introduced by Hörmander. As a reference see e.g. [10, Section 10.1]. A special case is the Fréchet space $\mathscr{E}(\Omega)$, i.e. the space $C^{\infty}(\Omega)$ equipped with its natural topology, induced by the seminorms

$$
p_{K, m}(f):=\max _{x \in K,|\alpha| \leq m}\left|\partial^{\alpha} f(x)\right|, \quad m \in \mathbb{N}_{0}, K \subset \Omega \text { compact. }
$$

More generally recall that $k: \mathbb{R}^{N} \rightarrow(0, \infty)$ is called a tempered weight function if there are constants $C>0$ and $m \in \mathbb{N}$ such that

$$
\forall \xi, \eta \in \mathbb{R}^{N}: \quad k(\xi+\eta) \leq(1+C|\xi|)^{m} k(\eta) .
$$

Typical examples of tempered weight functions are $k(\xi)=\left(1+|\xi|^{2}\right)^{s / 2}$, where $s$ is an arbitrary real number, or $\tilde{P}(\xi)=\left(\sum_{|\alpha| \geq 0}\left|\partial^{\alpha} P(\xi)\right|^{2}\right)^{1 / 2}$, where $P \in \mathbb{C}\left[X_{1}, \ldots, X_{N}\right]$ is a polynomial (see [10, Example 10.1.3]). 
For a tempered weight function $k$ and $1 \leq p<\infty$ let $B_{p, k}:=\left\{u \in \mathscr{S}^{\prime} ; \hat{u}\right.$ is a function and

$$
\left.\|u\|_{p, k}:=\left((2 \pi)^{-N} \int_{\mathbb{R}^{N}}|k(\xi) \hat{u}(\xi)|^{p} d \xi\right)^{1 / p}<\infty\right\} .
$$

Then $B_{p, k}$ together with the norm $\|\cdot\|_{p, k}$ is a Banach space (cf. [10, Theorem 10.1.7]).

Moreover, for $1 \leq p<\infty$ and a tempered weight function $k$ let

$$
B_{p, k}^{\text {loc }}(\Omega):=\left\{u \in \mathscr{D}^{\prime}(\Omega) ; \forall \phi \in \mathscr{D}(\Omega): \phi u \in B_{p, k}\right\} .
$$

$B_{p, k}^{\text {loc }}(\Omega)$ equipped with the family of seminorms $u \mapsto\|\phi u\|_{p, k}, \phi \in \mathscr{D}(\Omega)$, becomes a Fréchet space. For $p=2$ and $k(\xi)=\left(1+|\xi|^{2}\right)^{s / 2}, s \in \mathbb{R}$, one obtains in this way the local Sobolev space $\mathscr{H}_{(s)}^{\text {loc }}(\Omega)$ of order $s$.

Obviously, for any compact exhaustion $\left(K_{n}\right)_{n \in \mathbb{N}}$ of $\Omega$ and $\phi_{n} \in \mathscr{D}(\Omega)$ satisfying $K_{n} \subset\left\{\phi_{n}=1\right\}$ the topology of $B_{p, k}^{\text {loc }}(\Omega)$ is generated by the sequence of seminorms $u \mapsto\left\|\phi_{n} u\right\|_{p, k}, n \in \mathbb{N}$. Furthermore, $\mathscr{E}(\Omega) \subset B_{p, k}^{\text {loc }}(\Omega)$, and the inclusion is continuous and has dense range (cf. [10, Theorems 10.1.26 and 10.1.17]).

Finally, for a sequence $\left(p_{j}\right)_{j \in \mathbb{N}} \in[1, \infty)^{\mathbb{N}}$ and a sequence $\left(k_{j}\right)_{j \in \mathbb{N}}$ of tempered weight functions let $\bigcap_{j=1}^{\infty} B_{p_{j}, k_{j}}^{\text {loc }}(\Omega)$ be equipped with the family of seminorms $u \mapsto\|\varphi u\|_{j}:=\|\varphi u\|_{p_{j}, k_{j}}, j \in \mathbb{N}, \varphi \in \mathscr{D}(\Omega)$. With these seminorms $\bigcap_{j=1}^{\infty} B_{p_{j}, k_{j}}^{\text {loc }}(\Omega)$ is a Fréchet space whose topology is obviously generated by the increasing sequence of seminorms

$$
q_{n}(u):=\max _{1 \leq k, j \leq n}\left\|\phi_{k} u\right\|_{j}, \quad n \in \mathbb{N},
$$

with $\phi_{k}$ as above.

By the preceding remarks we have $\mathscr{E}(\Omega) \hookrightarrow \bigcap_{j=1}^{\infty} B_{p_{j}, k_{j}}^{\text {loc }}(\Omega)$ continuously with dense range (cf. [10, Theorem 10.1.17]). Since polynomials are dense in $\mathscr{E}(\Omega)$ (cf. [15, p. 160]), it now follows immediately that polynomials with coefficients in $\mathbb{Q}+i \mathbb{Q}$ are dense in $\bigcap_{j=1}^{\infty} B_{p_{j}, k_{j}}^{\text {loc }}(\Omega)$, so that $\bigcap_{j=1}^{\infty} B_{p_{j}, k_{j}}^{\text {loc }}(\Omega)$ is a separable Fréchet space for each open subset $\Omega \subset \mathbb{R}^{N}$.

For the special case $k_{j}(\xi)=(1+|\xi|)^{j}$ and arbitrary $1 \leq p_{j}<\infty$ one obtains $\mathscr{E}(\Omega)=\bigcap_{j=1}^{\infty} B_{p_{j}, k_{j}}^{\text {loc }}(\Omega)$ as Fréchet spaces (cf. [10, Remark following Theorem 10.1.26]).

3. The space of zero solutions. For a polynomial $P \in \mathbb{C}\left[X_{1}, \ldots, X_{N}\right]$ let $\tilde{P}(\xi)=\left(\sum_{|\alpha| \geq 0}\left|\partial^{\alpha} P(\xi)\right|^{2}\right)^{1 / 2}$. Then $\tilde{P}$ is a tempered weight function (see [10, Example 10.1.3]) and since real powers and products of tempered weight functions are again tempered weight functions (cf. [10, Theorem 10.1.4]), $k / \tilde{P}$ is a tempered weight function whenever so is $k$. 
By [10, Theorem 10.1.22 and its proof] the mapping

$$
P(D): B_{p, k}^{\mathrm{loc}}(\Omega) \rightarrow B_{p, k / \tilde{P}}^{\mathrm{loc}}(\Omega), \quad u \mapsto P(D) u,
$$

is continuous, where as usual $P(D) u=\sum_{|\alpha| \leq m}(-i)^{|\alpha|} a_{\alpha} \partial^{\alpha} u$ for $P(\xi)=$ $\sum_{|\alpha| \leq m} a_{\alpha} \xi^{\alpha}$. Therefore,

$$
P(D): \bigcap_{j=1}^{\infty} B_{p_{j}, k_{j}}^{\mathrm{loc}}(\Omega) \rightarrow \bigcap_{j=1}^{\infty} B_{p_{j}, k_{j} / \tilde{P}^{\mathrm{loc}}}(\Omega)
$$

is continuous, so that

$$
\mathcal{N}_{P,\left(p_{j}, k_{j}\right)}(\Omega):=\left\{u \in \bigcap_{j=1}^{\infty} B_{p_{j}, k_{j}}^{\mathrm{loc}}(\Omega) ; P(D) u=0\right\}
$$

is a closed subspace of the separable Fréchet space $\bigcap_{j=1}^{\infty} B_{p_{j}, k_{j}}^{\text {loc }}(\Omega)$, hence a separable Fréchet space itself. When it is clear from the context, we omit the reference to the sequences $\left(k_{j}\right)_{j \in \mathbb{N}}$ and $\left(p_{j}\right)_{j \in \mathbb{N}}$ and simply write $\mathcal{N}_{P}(\Omega)$ instead of $\mathcal{N}_{P,\left(p_{j}, k_{j}\right)}(\Omega)$. For the special case $k_{j}(\xi)=(1+|\xi|)^{j}$, that is, $\bigcap_{j=1}^{\infty} B_{p_{j}, k_{j}}^{\text {loc }}(\Omega)=\mathscr{E}(\Omega)$, we simply write $\mathscr{E}_{P}(\Omega)$ instead of $\mathcal{N}_{P,\left(p_{j}, k_{j}\right)}(\Omega)$, i.e. $\mathscr{E}_{P}(\Omega)$ is the vector space

$$
\left\{u \in C^{\infty}(\Omega) ; P(D) u=0\right\}
$$

equipped with the topology induced by the seminorms

$$
p_{K, m}(u):=\max _{x \in K,|\alpha| \leq m}\left|\partial^{\alpha} u(x)\right|, \quad m \in \mathbb{N}_{0}, K \subset \Omega \text { compact. }
$$

Obviously, $\mathscr{E}_{P}(\Omega) \subset \mathcal{N}_{P,\left(p_{j}, k_{j}\right)}(\Omega)$ for every $\left(p_{j}, k_{j}\right)_{j \in \mathbb{N}}$. Note that for a hypoelliptic polynomial $P$ one always has $\mathcal{N}_{P,\left(p_{j}, k_{j}\right)}(\Omega) \subset C^{\infty}(\Omega)$. Hence it follows from the continuity of the inclusion $\mathscr{E}(\Omega) \hookrightarrow \bigcap_{j=1}^{\infty} B_{p_{j}, k_{j}}^{\text {loc }}(\Omega)$ and the Open Mapping Theorem that $\mathcal{N}_{P,\left(p_{j}, k_{j}\right)}(\Omega)=\mathscr{E}_{P}(\Omega)$ as Fréchet spaces whenever $P$ is hypoelliptic.

In the special case when $N=2$ and $P(D)=\frac{1}{2}\left(\partial_{1}+i \partial_{2}\right)$ we find again, by the Open Mapping Theorem, that $\mathscr{E}_{P}(\Omega)$ is the space of holomorphic functions on $\Omega$ equipped with the compact-open topology.

We now introduce composition operators on $\mathcal{N}_{P,\left(p_{j}, k_{j}\right)}(\Omega)$. For two open subsets $\Omega_{1}$ and $\Omega_{2}$ of $\mathbb{R}^{N}$ and a diffeomorphism $f: \Omega_{1} \rightarrow \Omega_{2}$ there is a unique continuous linear mapping $f^{*}: \mathscr{D}^{\prime}\left(\Omega_{2}\right) \rightarrow \mathscr{D}^{\prime}\left(\Omega_{1}\right)$ such that $f^{*} u=$ $u \circ f$ if $u \in C\left(\Omega_{2}\right)$. For $\varphi \in \mathscr{D}\left(\Omega_{1}\right)$ one has $\left\langle f^{*} u, \varphi\right\rangle=\left\langle u,\left|\operatorname{det} J f^{-1}\right| \varphi \circ f^{-1}\right\rangle$, where $J f^{-1}$ denotes the Jacobian of $f^{-1}$. Moreover, $(g \circ f)^{*} u=f^{*} g^{*} u$ for a second diffeomorphism $g$ from $\Omega_{2}$ to $\Omega_{3}$ and every distribution $u$ on $\Omega_{3}$ (see e.g. [10, Section 6.1]). We sometimes use the notation $u(f)$ or $u \circ f$ instead of $f^{*} u$.

Note that for $\phi \in \mathcal{E}\left(\Omega_{2}\right)$ one has

$$
\left\langle f^{*}(\phi u), \varphi\right\rangle=\left\langle u,\left|\operatorname{det} J f^{-1}\right|\left(\phi \circ f \circ f^{-1}\right)\left(\varphi \circ f^{-1}\right)\right\rangle=\left\langle\left(f^{*} \phi\right)\left(f^{*} u\right), \varphi\right\rangle
$$

for every $\varphi \in \mathscr{D}\left(\Omega_{1}\right)$, i.e. $f^{*}(\phi u)=\left(f^{*} \phi\right)\left(f^{*} u\right)$. 
A simple property of $f^{*}$ is stated in the next proposition.

Proposition 3.1. Let $f: \Omega_{1} \rightarrow \Omega_{2}$ be a diffeomorphism. For $u \in$ $\mathscr{D}^{\prime}\left(\Omega_{2}\right)$ one has $\operatorname{supp} f^{*} u=f^{-1}(\operatorname{supp} u)$.

Proof. Let $V$ be an open superset of $\Omega_{2} \backslash \operatorname{supp} u$, i.e. $\langle u, \varphi\rangle=0$ for all $\varphi \in \mathscr{D}(V)$. If $\psi \in \mathscr{D}\left(f^{-1}(V)\right)$, then clearly $\psi \circ f^{-1} \in \mathscr{D}(V)$, hence $\left|\operatorname{det} J f^{-1}\right|\left(\psi \circ f^{-1}\right) \in \mathscr{D}(V)$, so that

$$
\left\langle f^{*} u, \psi\right\rangle=\left\langle u,\left|\operatorname{det} J f^{-1}\right|\left(\psi \circ f^{-1}\right)\right\rangle=0 .
$$

Because $f^{-1}(V)$ is open it follows that $\operatorname{supp} f^{*} u \subset \Omega_{1} \backslash f^{-1}(V)$. Since $V$ was an arbitrary open subset of $\Omega_{2} \backslash \operatorname{supp} u$ it follows that

$$
\begin{aligned}
\operatorname{supp} f^{*} u & \subset \bigcap_{V \subset \Omega_{2} \backslash \operatorname{supp} u, V \text { open }} \Omega_{1} \backslash f^{-1}(V)=\Omega_{1} \backslash f^{-1}\left(\bigcup_{V \subset \Omega_{2} \backslash \operatorname{supp} u, V \text { open }} V\right) \\
& =\Omega_{1} \backslash f^{-1}\left(\Omega_{2} \backslash \operatorname{supp} u\right)=f^{-1}(\operatorname{supp} u) .
\end{aligned}
$$

Applying to the diffeomorphism $f^{-1}$ what has been shown so far, we also see

$$
\operatorname{supp} u=\operatorname{supp}\left(f^{-1}\right)^{*} f^{*} u \subset f\left(\operatorname{supp} f^{*} u\right),
$$

i.e. $f^{-1}(\operatorname{supp} u) \subset \operatorname{supp} f^{*} u$, too.

We are interested in diffeomorphisms which respect the kernel of a given differential operator on $\bigcap_{j=1}^{\infty} B_{p_{j}, k_{j}}^{\text {loc }}\left(\Omega_{2}\right)$. This is expressed by the following notion.

Definition 3.2. Let $\Omega_{1}, \Omega_{2} \subset \mathbb{R}^{N}$ be open, $\left(p_{j}\right)_{j \in \mathbb{N}} \in[1, \infty)^{\mathbb{N}},\left(k_{j}\right)_{j \in \mathbb{N}}$ be a sequence of tempered weight functions, and let $f: \Omega_{1} \rightarrow \Omega_{2}$ be a diffeomorphism. A polynomial $P$ is called $\left(p_{j}, k_{j}\right)_{j \in \mathbb{N}^{-}} f$-invariant (or simply $f$-invariant) if for every $u \in \mathscr{D}^{\prime}\left(\Omega_{2}\right)$ one has $u \in \mathcal{N}_{P,\left(p_{j}, k_{j}\right)}\left(\Omega_{2}\right)$ if and only if $u \circ f \in \mathcal{N}_{P,\left(p_{j}, k_{j}\right)}\left(\Omega_{1}\right)$.

REMARK 3.3. (i) Obviously, $P$ is $f$-invariant if and only if $P$ is $f^{-1}$ invariant. Moreover, if $\Omega_{1}=\Omega_{2}$, for given $P$ the set of diffeomorphisms $f$ on $\Omega_{1}$ for which $P$ is $\left(p_{j}, k_{j}\right)_{j \in \mathbb{N}^{-}} f$-invariant forms a group under composition.

(ii) Since the translations $\tau_{b}(x)=x+b$ commute with $P(D)$ and $\mathcal{F}\left(u \circ \tau_{b}\right)(\xi)=e^{i\langle b, \xi\rangle} \mathcal{F}(u)(\xi)$ it follows that $P$ is $\tau_{b}$-invariant for every $b \in \mathbb{R}^{N}$.

(iii) Because $\mathscr{E}_{P}\left(\Omega_{2}\right) \subset \mathcal{N}_{P}\left(\Omega_{2}\right)$ it is necessary for the $f$-invariance of $P$ that for $\varphi \in C^{\infty}\left(\Omega_{2}\right)$ one has $P(D) \varphi=0$ if and only if $P(D)(\varphi \circ f)$ $=0$. Therefore, if $\mathcal{N}_{P,\left(p_{j}, k_{j}\right)}\left(\Omega_{2}\right)=\mathscr{E}_{P}\left(\Omega_{2}\right)$ (which holds in particular when $k_{j}(\xi)=(1+|\xi|)^{j}, j \in \mathbb{N}$, or when $P$ is hypoelliptic), the aforementioned necessary condition is also sufficient for $\left(p_{j}, k_{j}\right)_{j \in \mathbb{N}^{-}} f$-invariance of $P$.

(iv) For a given polynomial $P$ the conditions on $f=\left(f_{1}, \ldots, f_{N}\right)$ ensuring that $P$ is $f$-invariant can be quite restrictive. Since exponential solutions of $P(D) u=0$, i.e. solutions of the form $u(x)=Q(x) \exp (i\langle\zeta, x\rangle)$ where $Q$ is a polynomial and $\zeta \in \mathbb{C}^{N}$ is a root of $P$, always belong to $\mathcal{N}_{P}(\Omega)$, one can 
derive certain differential equations which have to be satisfied by $f$ in order that $P$ is $f$-invariant.

For example, if $P$ is a polynomial of degree $2, P(\zeta)=\sum_{j, k=1}^{N} a_{j, l} \zeta_{j} \zeta_{k}+$ $\sum_{j=1}^{N} b_{j} \zeta_{j}+c$, and one defines for $1 \leq j, k \leq N$ the differential operators

$$
B_{j}(\zeta, f):=\sum_{m=1}^{N} \zeta_{m} \partial_{j} f_{m}
$$

and

$$
A_{j, k}(\zeta, f):=\sum_{m, l=1}^{N} \zeta_{m} \zeta_{l} \partial_{j} f_{m} \partial_{k} f_{l}-i \sum_{m=1}^{N} \zeta_{m} \partial_{j} \partial_{k} f_{m},
$$

then $f$ has to satisfy (by taking $Q \equiv 1$ ) the (non-linear!) differential equations

$$
\begin{aligned}
\forall \zeta \in\left\{z \in \mathbb{C}^{N} ; P(z)=\right. & 0\}: \\
& \sum_{j, k=1}^{N} a_{j, k} A_{j, k}(\zeta, f)+\sum_{j=1}^{N} b_{j} B_{j}(\zeta, f)+c=0 .
\end{aligned}
$$

To end this section we give characterizations of those $f$ such that certain important polynomials are $f$-invariant.

Proposition 3.4. Let $f: \Omega_{1} \rightarrow \Omega_{2}$ be a diffeomorphism. If $P$ is a $\left(p_{j}, k_{j}\right)_{j \in \mathbb{N}^{-}} f$-invariant polynomial then $f^{*}: \mathcal{N}_{P,\left(p_{j}, k_{j}\right)}\left(\Omega_{2}\right) \rightarrow \mathcal{N}_{P,\left(p_{j}, k_{j}\right)}\left(\Omega_{1}\right)$ is a topological isomorphism.

Proof. By the $f$-invariance of $P$ the mapping is well-defined and obviously linear. From the continuity of $\bigcap_{j=1}^{\infty} B_{p_{j}, k_{j}}^{\text {loc }}\left(\Omega_{2}\right) \hookrightarrow \mathscr{D}^{\prime}\left(\Omega_{2}\right)$ and $f^{*}$ : $\mathscr{D}^{\prime}\left(\Omega_{2}\right) \rightarrow \mathscr{D}^{\prime}\left(\Omega_{1}\right)$ the continuity of $f^{*}$ follows immediately by the Closed Graph Theorem for Fréchet spaces. Since $f^{*}$ is obviously one-to-one and onto, the Open Mapping Theorem for Fréchet spaces gives the result.

Recall that for $\phi \in \mathscr{E}(\Omega)$ and $u \in \mathscr{D}^{\prime}(\Omega)$ one has $f^{*}(\phi u)=f^{*}(\phi) f^{*}(u)$. Moreover, recall that the topology on $\mathcal{N}_{P,\left(p_{j}, k_{j}\right)}(\Omega)$ is generated by the increasing sequence of seminorms $q_{n}(u):=\max _{1 \leq l, j \leq n}\left\|\varphi_{l} u\right\|_{j}, n \in \mathbb{N}$, where $\|\varphi u\|_{j}=\|\varphi u\|_{p_{j}, k_{j}}$ and $\left(\varphi_{n}\right)_{n \in \mathbb{N}} \in \mathscr{D}(\Omega)^{\mathbb{N}}$ satisfies $\operatorname{supp} \varphi_{n} \subset K_{n+1} \subset$ $\left\{\varphi_{n+1}=1\right\}, n \in \mathbb{N}$, for a compact exhaustion $\left(K_{n}\right)_{n \in \mathbb{N}}$ of $\Omega$.

Corollary 3.5. Let $\Omega \subset \mathbb{R}^{N}$ be open, $\Omega_{1} \subset \Omega$ open, and $f: \Omega \rightarrow$

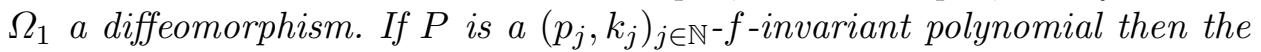
mapping

$$
\mathcal{N}_{P,\left(p_{j}, k_{j}\right)}(\Omega) \rightarrow \mathcal{N}_{P,\left(p_{j}, k_{j}\right)}(\Omega), \quad u \mapsto u_{\Omega_{\Omega_{1}}} \circ f,
$$

is linear and continuous. We denote it again by $f^{*}$ and sometimes also write $u \circ f$ instead of $f^{*} u$. With this notation $\operatorname{supp} f^{*} u=f^{-1}\left(\operatorname{supp} u \cap \Omega_{1}\right)$. 
Moreover,

$$
\forall n \in \mathbb{N}: \quad q_{n}\left(f^{*} u\right) \leq \max _{1 \leq l \leq n} q_{n+1}\left(f^{*}\left[\left(\varphi_{l} \circ f^{-1}\right) u\right]\right) .
$$

Proof. The continuity follows immediately from the obvious continuity of the restriction map $\mathcal{N}_{P}(\Omega) \rightarrow \mathcal{N}_{P}\left(\Omega_{1}\right), u \mapsto u_{\mid \Omega_{1}}$, and Proposition 3.4 . while supp $f^{*} u=f^{-1}\left(\operatorname{supp} u \cap \Omega_{1}\right)$ is a direct consequence of Proposition 3.1

Finally, because $\operatorname{supp} \varphi_{l} \subset K_{l+1} \subset\left\{\varphi_{l+1}=1\right\}$ we have

$$
\varphi_{l+1} f^{*}\left[\left(\varphi_{l} \circ f^{-1}\right) u\right]=\varphi_{l+1} f^{*}\left(\varphi_{l} \circ f^{-1}\right) f^{*}(u)=\varphi_{l+1} \varphi_{l} f^{*}(u)=\varphi_{l} f^{*}(u),
$$

so that

$$
\begin{aligned}
q_{n}\left(f^{*} u\right) & =\max _{1 \leq l, j \leq n}\left\|\varphi_{l} f^{*}(u)\right\|_{j}=\max _{1 \leq l, j \leq n}\left\|\varphi_{l+1} f^{*}\left[\left(\varphi_{l} \circ f^{-1}\right) u\right]\right\|_{j} \\
& \leq \max _{1 \leq l, j \leq n} q_{n+1}\left(\varphi_{l+1} f^{*}\left[\left(\varphi_{l} \circ f^{-1}\right) u\right]\right)=\max _{1 \leq l \leq n} q_{n+1}\left(f^{*}\left[\left(\varphi_{l} \circ f^{-1}\right) u\right]\right) .
\end{aligned}
$$

We now give characterizations of those $f$ such that certain important polynomials $P$ are $f$-invariant. We are sure that these are known. Nevertheless, since we could not find a reference, we give the proofs for the sake of completeness. To formulate the next proposition more conveniently, we write $x=\left(x^{\prime}, x_{N}\right)$ with $x^{\prime} \in \mathbb{R}^{N-1}$ and $x_{N} \in \mathbb{R}$ for $x=\left(x_{1}, \ldots, x_{N}\right) \in \mathbb{R}^{N}$.

Proposition 3.6.

(a) Let $N=2$ and $P(\xi)=\frac{1}{2}\left(i \xi_{1}-\xi_{2}\right)$, i.e. $P(D)=\bar{\partial}$ is the CauchyRiemann operator. Then $P$ is $f$-invariant if and only if $\bar{\partial} f=0$, i.e. $f$ is holomorphic.

(b) Let $P(\xi)=-|\xi|^{2}$, i.e. $P(D)=\Delta$ is the Laplacian. Then $P$ is $f$ invariant if and only if the following conditions hold.

(i) $\Delta f_{j}=0$ for all $1 \leq j \leq N$.

(ii) $\left|\nabla f_{j}\right|=\left|\nabla f_{k}\right|$ and $\left\langle\nabla f_{j}, \nabla f_{k}\right\rangle=0$ for all $1 \leq j \neq k \leq N$,

that is, the Jacobian of $f$ is a multiple of an orthogonal matrix at each point.

(c) On $\mathbb{R}^{N+1}$ let $P(\xi)=\left|\xi^{\prime}\right|^{2}+i \xi_{N+1}$, i.e. $P(D)$ is the heat operator $H=\Delta_{x^{\prime}}-\partial_{N+1}$, where the $(N+1)$ th variable is considered as time and $\Delta_{x^{\prime}}$ denotes the Laplacian with respect to the first $N$ variables. Then $P$ is $f$-invariant if and only if

$$
f(x)=\left(\alpha A x^{\prime}, \alpha^{2} x_{N+1}\right)+b
$$

where $\alpha \in \mathbb{R} \backslash\{0\}, b \in \mathbb{R}^{N+1}$, and $A \in \mathbb{R}^{N \times N}$ is an orthogonal matrix.

(d) On $\mathbb{R}^{N+1}$ let $P(\xi)=\xi_{N+1}^{2}-\left|\xi^{\prime}\right|^{2}$, i.e. $P(D)$ is the wave operator $\square=\Delta_{x^{\prime}}-\partial_{N+1}^{2}$, where again the $(N+1)$ th variable is considered as time and $\Delta_{x^{\prime}}$ denotes the Laplacian with respect to the first $N$ 
variables. If all tempered weight functions $k_{j}$ are radial functions and one requires $\partial_{k} f_{N+1}=\partial_{N+1} f_{k}=0$ for all $1 \leq k \leq N$ (i.e. via the transformation $f$ the time variable has no influence on the space variables and vice versa), then $P$ is $f$-invariant if and only if

$$
f(x)=\alpha\left(A x^{\prime}, x_{N+1}\right)+b
$$

where $\alpha \in \mathbb{R} \backslash\{0\}, b \in \mathbb{R}^{N+1}$ and $A \in \mathbb{R}^{N \times N}$ is an orthogonal matrix.

Proof. Since the polynomials in (a)-(c) are hypoelliptic, in these cases $f$-invariance of $P$ means that for $u \in C^{\infty}\left(\Omega_{2}\right)$ one has $P(D) u=0$ if and only if $P(D)(u \circ f)=0$. Now (a) follows after a short calculation from the Cauchy-Riemann equations.

In case of (b), taking $Q(x)=x_{j}$ and $\zeta=0$, the corresponding exponential solution $Q(x) \exp (i\langle\zeta, x\rangle)$ shows that $\Delta f_{j}=0,1 \leq j \leq N$, is necessary for $P$ to be $f$-invariant. With this, the necessary conditions (1) above turn into

$$
\forall \zeta \in\left\{z \in \mathbb{C}^{N} ; P(z)=0\right\}: \quad 0=-\sum_{m, l=1}^{N} \zeta_{m} \zeta_{l}\left\langle\nabla f_{m}, \nabla f_{l}\right\rangle .
$$

For $1 \leq j \neq k \leq N$ set $\zeta_{j}=1, \zeta_{k}=i$ and $\zeta_{l}=0$ for $l \notin\{j, k\}$. Then $\zeta$ is a root of $P$ and hence

$$
\forall 1 \leq j \neq k \leq N: \quad 0=\left|\nabla f_{k}\right|^{2}-\left|\nabla f_{j}\right|^{2}-2 i\left\langle\nabla f_{j}, \nabla f_{k}\right\rangle
$$

is necessary, which gives necessity of conditions (i) and (ii).

On the other hand, a straightforward calculation gives, for $u \in C^{\infty}\left(\Omega_{2}\right)$,

$$
\Delta(u \circ f)=\sum_{j=1}^{N}\left(\Delta f_{j}\right)\left(\left(\partial_{j} u\right) \circ f\right)+\sum_{j, k=1}^{N}\left(\left(\partial_{j} \partial_{k} u\right) \circ f\right)\left\langle\nabla f_{j}, \nabla f_{k}\right\rangle .
$$

Therefore, if $f$ satisfies (i) and (ii) we have, for all $u \in C^{\infty}\left(\Omega_{2}\right)$,

$$
\Delta(u \circ f)=\left|\nabla f_{1}\right|^{2}((\Delta u) \circ f) .
$$

Since $f$ is a diffeomorphism, we have $\left|\nabla f_{1}\right|^{2} \neq 0$ everywhere, so it follows that $\Delta u=0$ if and only if $\Delta(u \circ f)=0$, so that (i) and (ii) are also sufficient for the $f$-invariance of $P$.

To show necessity in (c), assume that $P$ is $f$-invariant. Take $Q(x)=x_{j}$ for $1 \leq j \leq N$ and $\zeta=0$. From the corresponding exponential solutions it follows that $H f_{j}=0$ for $1 \leq j \leq N$. Therefore, the necessary conditions (1) become

$$
\begin{aligned}
\forall \zeta \in\left\{z \in \mathbb{C}^{N} ; P(z)=0\right\}: \quad 0 & =i B_{N+1}(\zeta, f)+\sum_{j=1}^{N} A_{j, j}(\zeta, f) \\
& =i \zeta_{N+1} H f_{N+1}+\sum_{l, m=1}^{N+1} \zeta_{l} \zeta_{m}\left\langle\nabla_{x^{\prime}} f_{l}, \nabla_{x^{\prime}} f_{m}\right\rangle
\end{aligned}
$$


where $\nabla_{x^{\prime}} f_{j}:=\left(\partial_{1} f_{j}, \ldots, \partial_{N} f_{j}\right)$ denotes the gradient of $f_{j}$ with respect to the space variables $x^{\prime}$.

Taking $\zeta_{j}=1$ for a fixed $j \in\{1, \ldots, N\}, \zeta_{N+1}=i$ and $\zeta_{l}=0$ for $l \notin\{j, N+1\}$ we get a root of $P$ giving

$$
0=-H f_{N+1}-\left|\nabla_{x^{\prime}} f_{N+1}\right|^{2}+\left|\nabla_{x^{\prime}} f_{j}\right|^{2}+2 i\left\langle\nabla_{x^{\prime}} f_{j}, \nabla_{x^{\prime}} f_{N+1}\right\rangle,
$$

so that

$$
\forall 1 \leq j \leq N: \quad H f_{N+1}+\left|\nabla_{x^{\prime}} f_{N+1}\right|^{2}=\left|\nabla_{x^{\prime}} f_{j}\right|^{2},\left\langle\nabla_{x^{\prime}} f_{j}, \nabla_{x^{\prime}} f_{N+1}\right\rangle=0 .
$$

On the other hand, taking $\zeta_{N+1}=1$, for fixed $j \in\{1, \ldots, N\}$ any square $\operatorname{root} \zeta_{j}=\sqrt{i}$ of $i$, and $\zeta_{l}=0$ for $l \notin\{j, N+1\}$ gives another root of $P$ which yields

$$
0=i H f_{N+1}+\left|\nabla_{x^{\prime}} f_{N+1}\right|^{2}+i\left|\nabla_{x^{\prime}} f_{j}\right|^{2}+2 \sqrt{i}\left\langle\nabla_{x^{\prime}} f_{N+1}, \nabla_{x^{\prime}} f_{j}\right\rangle,
$$

so that $\left|\nabla_{x^{\prime}} f_{N+1}\right|^{2}=0$, i.e. $f_{N+1}$ only depends on $x_{N+1}$.

From the last two sets of equations it follows that $\left|\nabla_{x^{\prime}} f_{j}\right|^{2}=H f_{N+1}=$ $\partial_{N+1} f_{N+1}$ for all $1 \leq j \leq N$. Because $f_{N+1}$ only depends on $x_{N+1}$ it follows that $\Delta_{x^{\prime}} f_{j}=0$ for all $1 \leq j \leq N$, so that in addition $0=H f_{j}=\partial_{N+1} f_{j}$ for all $1 \leq j \leq N$. Therefore, $f_{j}$ does not depend on $x_{N+1}$ for $1 \leq j \leq N$. Since $f_{N+1}$ does not depend on $x^{\prime}$ and $\left|\nabla_{x^{\prime}} f_{j}\right|^{2}=\partial_{N+1} f_{N+1}$ it follows that $\nabla_{x^{\prime}} f_{j}$ as well as $\partial_{N+1} f_{N+1}$ are constant, i.e. $f_{j}$ is an affine function of $x^{\prime}$ and $f_{N+1}$ is an affine function of $x_{N+1}$.

This means that there are $\gamma, \beta \in \mathbb{R}, a \in \mathbb{R}^{N}$ and $B \in \mathbb{R}^{N \times N}$ such that

$$
\forall x \in \Omega_{1}: \quad f\left(x^{\prime}, x_{N+1}\right)=\left(B x^{\prime}+a, \gamma x_{N+1}+\beta\right) .
$$

Since $f$ is a diffeomorphism and $\left|\nabla_{x^{\prime}} f_{j}\right|^{2}=\partial_{N+1} f_{N+1}$ we have $\gamma>0$ and $B$ has to be invertible.

To see that $B$ is actually a multiple of an orthogonal matrix, fix $1 \leq j \neq$ $k \leq N$ and set $\zeta_{j}=1, \zeta_{k}=i$ and $\zeta_{l}=0$ for $l \notin\{j, k\}$. This gives another root of $P$ yielding the equation

$$
0=\left|\nabla_{x^{\prime}} f_{j}\right|^{2}-\left|\nabla_{x^{\prime}} f_{k}\right|^{2}+2 i\left\langle\nabla_{x^{\prime}} f_{j}, \nabla_{x^{\prime}} f_{k}\right\rangle
$$

so that

$$
\forall 1 \leq j \neq k \leq N: \quad\left|\nabla_{x^{\prime}} f_{j}\right|=\left|\nabla_{x^{\prime}} f_{k}\right|,\left\langle\nabla_{x^{\prime}} f_{j}, \nabla_{x^{\prime}} f_{k}\right\rangle=0,
$$

which means, since $\left|\nabla_{x^{\prime}} f_{j}\right|^{2}=\partial_{N+1} f_{N+1}=\gamma>0$, that $A:=(1 / \sqrt{\gamma}) B$ is orthogonal.

This shows that the condition on $f$ stated in (b) is necessary for $P$ to be $f$-invariant.

To show its sufficiency as well, observe that by a straightforward calculation for $f$ of the stated form one obtains

$$
\forall u \in C^{\infty}\left(\Omega_{2}\right): \quad H(u \circ f)=\alpha^{2}((H u) \circ f)
$$

so that indeed $H u=0$ if and only if $H(u \circ f)=0$. 
In order to prove (d) we assume that all tempered weight functions are radial and that $\partial_{k} f_{N+1}=\partial_{N+1} f_{k}=0$ for all $1 \leq k \leq N$. To prove necessity assume that $P$ is $f$-invariant. Taking $Q(x)=x_{j}$ and $\zeta=0$ it follows from the corresponding exponential solution that $\square f_{j}=0$ for all $1 \leq j \leq N+1$. Since by assumption $f_{N+1}$ is independent of $x^{\prime}$ it follows that $0=\square f_{N+1}=\partial_{N+1}^{2} f_{N+1}$ so that $f_{N+1}$ has to be an affine function of $x_{N+1}$, i.e. there are $\alpha, \beta \in \mathbb{R}$ such that $f_{N+1}\left(x^{\prime}, x_{N+1}\right)=\alpha x_{N+1}+\beta$.

With this, the necessary conditions (1) turn into $\forall \zeta \in\left\{z \in \mathbb{C}^{N} ; P(z)=0\right\}: 0=\sum_{l, m=1}^{N+1} \zeta_{l} \zeta_{m}\left(\left\langle\nabla_{x^{\prime}} f_{l}, \nabla_{x^{\prime}} f_{m}\right\rangle-\partial_{N+1} f_{l} \partial_{N+1} f_{m}\right)$. For fixed $1 \leq j \neq k \leq N$ set $\zeta_{j}=1, \zeta_{k}=i$ and $\zeta_{l}=0$ for $l \notin\{j, k\}$ so that $\zeta$ is a root of $P$ giving

$$
0=\left|\nabla_{x^{\prime}} f_{j}\right|^{2}-\left|\nabla_{x^{\prime}} f_{k}\right|^{2}+2 i\left\langle\nabla_{x^{\prime}} f_{j}, \nabla_{x^{\prime}} f_{k}\right\rangle,
$$

so that

$$
\forall 1 \leq j \neq k \leq N: \quad\left|\nabla_{x^{\prime}} f_{j}\right|=\left|\nabla_{x^{\prime}} f_{k}\right|,\left\langle\nabla_{x^{\prime}} f_{j}, \nabla_{x^{\prime}} f_{k}\right\rangle=0 .
$$

On the other hand, for $1 \leq j \leq N$ fixed, let $\zeta_{j}=\zeta_{N+1}=1$ and $\zeta_{l}=0$ for $l \notin\{j, N+1\}$ so that $\zeta$ is a root of $P$ yielding

$$
\forall 1 \leq j \leq N: \quad 0=\left|\nabla_{x^{\prime}} f_{j}\right|^{2}-\left(\partial_{N+1} f_{N+1}\right)^{2}=\left|\nabla_{x^{\prime}} f_{j}\right|^{2}-\alpha^{2} .
$$

In particular $\nabla_{x^{\prime}} f_{j}$ is a constant function for all $1 \leq j \leq N$, independent of $x_{N+1}$ by hypothesis, i.e. $f_{j}$ is an affine function of $x^{\prime}$. Because $\alpha^{2}=\left|\nabla_{x^{\prime}} f_{j}\right|^{2}$ and $\left\langle\nabla_{x^{\prime}} f_{j}, \nabla_{x^{\prime}} f_{k}\right\rangle=0$ for all $1 \leq j \neq k \leq N$ there are $b^{\prime} \in \mathbb{R}^{N}$ and an orthogonal matrix $A \in \mathbb{R}^{N \times N}$ such that $\left(f_{1}, \ldots, f_{N}\right)\left(x^{\prime}, x_{N+1}\right)=\alpha A x^{\prime}+b^{\prime}$, proving the necessity.

In order to prove the sufficiency, recall that for $b \in \mathbb{R}^{N+1}$ and invertible $B \in \mathbb{R}^{(N+1) \times(N+1)}$ one has $\mathcal{F}(u \circ B)(\xi)=\left|\operatorname{det} B^{-1}\right| \mathcal{F}(u)\left(\left(B^{t}\right)^{-1} \xi\right)$, where $B^{t}$ denotes the transpose of $B$, as well as $\mathcal{F}\left(u \circ \tau_{b}\right)(\xi)=e^{i\langle b, \xi\rangle} \mathcal{F}(u)(\xi)$.

Since $k_{j}$ is a tempered weight function, there are $C>0$ and $m \in \mathbb{N}$ such that $k_{j}(\xi+\eta) \leq(1+C|\xi|)^{m} k_{j}(\eta)$ for all $\xi, \eta \in \mathbb{R}^{N+1}$. This yields

$$
\forall \xi \in \mathbb{R}^{N+1}: \quad k_{j}(0)(1+C|\xi|)^{-m} \leq k_{j}(\xi) \leq k_{j}(0)(1+C|\xi|)^{m} .
$$

Since $k_{j}$ is supposed to be a radial function and $A$ is orthogonal, it follows that $k(\xi)=k\left(A \xi^{\prime}, \xi_{N+1}\right)$. Using this we obtain, for $\phi \in \mathscr{D}\left(\Omega_{2}\right)$,

$$
\begin{aligned}
\int_{\mathbb{R}^{N+1}} \mid k_{j}(\xi) & \left.\mathcal{F}(\phi(u \circ f))(\xi)\right|^{p_{j}} d \xi \\
& =\int_{\mathbb{R}^{N+1}}\left|k_{j}(\xi) \alpha^{-(N+1)} \mathcal{F}\left(\left(\phi \circ f^{-1}\right) u\right)\left(\alpha^{-1}\left(A \xi^{\prime}, \xi_{N+1}\right)\right)\right|^{p_{j}} d \xi \\
& =\int_{\mathbb{R}^{N+1}}\left|k_{j}(\xi) \mathcal{F}\left(\left(\phi \circ f^{-1}\right) u\right)(\xi)\right|^{p_{j}}\left(\frac{k(\alpha \xi)}{k(\xi)}\right)^{p_{j}} d \xi
\end{aligned}
$$


Since $k(\alpha \xi) / k(\xi) \leq \max \{1,|\alpha|\}$ and since $f^{-1}$ is of the same form as $f$, it follows that $u \in \bigcap_{j=1}^{\infty} B_{p_{j}, k_{j}}^{\text {loc }}\left(\Omega_{2}\right)$ if and only if $u \circ f \in \bigcap_{j=1}^{\infty} B_{p_{j}, k_{j}}^{\text {loc }}\left(\Omega_{1}\right)$. Using again that $f^{-1}$ is of the same form as $f$, it is straightforward to show that

$$
\forall u \in \mathscr{D}^{\prime}(\Omega): \quad \square(u \circ f)=\alpha^{2}(\square u) \circ f .
$$

This finally shows that $P$ is indeed $f$-invariant.

4. Universal zero solutions. In this section we give a sufficient condition for a sequence of diffeomorphisms $f_{m}: \Omega \rightarrow \Omega_{m} \subset \Omega, m \in \mathbb{N}$, to have $\left(f_{m}^{*}\right)$-universal elements in $\mathcal{N}_{P}(\Omega)$. We first introduce the following notion.

Definition 4.1. Let $\Omega_{1} \subset \Omega$ be open subsets of $\mathbb{R}^{N}, P$ be a nonconstant polynomial, $\left(p_{j}\right)_{j \in \mathbb{N}} \in[1, \infty)^{\mathbb{N}}$, and $\left(k_{j}\right)_{j \in \mathbb{N}}$ a sequence of tempered weight functions. We say that $\Omega_{1}$ is $P$-approximable in $\Omega$ if $\left\{u_{\mid \Omega_{1}} ; u \in\right.$ $\left.\mathcal{N}_{P,\left(p_{j}, k_{j}\right)}(\Omega)\right\}$ is dense in $\mathcal{N}_{P,\left(p_{j}, k_{j}\right)}\left(\Omega_{1}\right)$. Again, if there is no danger of confusion we omit the reference to $\left(p_{j}\right)_{j \in \mathbb{N}} \in[1, \infty)^{\mathbb{N}}$ and $\left(k_{j}\right)_{j \in \mathbb{N}}$.

As is usually the case, the heart of our universality result is an approximation theorem. In our case it is the following theorem due to L. Hörmander. Recall that for an arbitrary subset $A$ of $\mathbb{R}^{N}, \mathscr{E}^{\prime}(A)$ denotes the space of distributions on $\mathbb{R}^{N}$ having compact support contained in $A$.

Theorem 4.2 ([10, Theorem 10.5.2]). Let $P$ be a non-constant polynomial, and $\Omega_{1} \subset \Omega$ open subsets of $\mathbb{R}^{N}$. Assume that for every $\mu \in \mathscr{E}^{\prime}(\bar{\Omega})$ the inclusion $\operatorname{supp} P(-D) \mu \subset \Omega_{1}$ implies $\operatorname{supp} \mu \subset \Omega_{1}$. Then $\Omega_{1}$ is $P$ approximable in $\Omega$.

In general, $P$-convexity for supports of neither $\Omega$ nor $\Omega_{1}$ is sufficient for $\Omega_{1}$ to be $P$-approximable in $\Omega$. For example, let $\Omega$ be any open subset of $\mathbb{R}^{2}$ containing the unit disk, $\Omega_{1}=\{x \in \Omega ;|x|>1 / 2\}$, and let $P(D)=$ $\frac{1}{2}\left(\partial_{1}+i \partial_{2}\right)$. Then $\mathscr{E}_{P}(\Omega)$ consists of the holomorphic functions in $\Omega$, and $P(D)$ being elliptic, $\Omega$ as well as $\Omega_{1}$ are $P$-convex for supports. However, $z=x_{1}+i x_{2} \mapsto 1 / z$ obviously belongs to $\mathscr{E}_{P}\left(\Omega_{1}\right)$ but not to the closure of $\left\{u_{\mid \Omega_{1}} ; u \in \mathscr{E}_{P}(\Omega)\right\}$ in $\mathscr{E}_{P}(\Omega)$.

The next proposition gives a simple sufficient condition for $P$-approximability.

Proposition 4.3. Let $\Omega \subset \mathbb{R}^{N}$ be open and $P$ a non-constant polynomial.

(i) If $\Omega_{1} \subset \Omega$ has convex components then $\Omega_{1}$ is $P$-approximable in $\Omega$.

(ii) Let $\Omega_{m} \subset \Omega$ be open subsets of $\Omega$ such that $\overline{\Omega_{m}} \cap \overline{\bigcup_{n \neq m} \Omega_{n}}=\emptyset$. Assume that for every $m$ and $u \in \mathscr{E}^{\prime}(\bar{\Omega})$ the inclusion $\operatorname{supp} P(-D) u \subset$ $\Omega_{m}$ implies $u \in \mathscr{E}^{\prime}\left(\Omega_{m}\right)$. Then $\bigcup_{m} \Omega_{m}$ is P-approximable in $\Omega$. 
Proof. (i) follows immediately from Theorem 4.2 and by applying to each component of $\Omega_{1}$ the fact that $\operatorname{ch} \operatorname{supp} u=\operatorname{ch} \operatorname{supp} P(-D) u$ where $\operatorname{ch} K$ denotes the closed convex hull of a set $K$ (cf. [10, Theorem 7.3.2]).

(ii) We show that the hypothesis of Theorem 4.2 is satisfied with $\bigcup_{m} \Omega_{m}$ in place of $\Omega_{1}$. Let $u \in \mathscr{E}^{\prime}(\bar{\Omega})$ with $\operatorname{supp} P(-D) u \subset \bigcup_{m} \Omega_{m}$. Set $f:=$ $P(-D) u$. Then, by compactness of $\operatorname{supp} f$, there is $r \in \mathbb{N}$ such that $\operatorname{supp} f \subset$ $\bigcup_{l=1}^{r} \Omega_{m_{l}}$. Let $\varphi_{1}, \ldots, \varphi_{r} \in \mathscr{E}(\Omega)$ satisfy $\Omega_{m_{l}} \subset\left\{\varphi_{l}=1\right\}$ and $\operatorname{supp} \varphi_{l} \cap$ $\operatorname{supp} \varphi_{k}=\emptyset$ for all $1 \leq l \neq k \leq r$.

For $\phi \in \mathscr{D}\left(\Omega_{m_{l}}\right)$ it follows that $D^{\alpha} \varphi_{l}=0$ in a neighbourhood of $\operatorname{supp} \phi$ for all $\alpha \neq 0$, so that by Leibniz' formula,

$$
\langle f, \phi\rangle=\left\langle\varphi_{l} f, \phi\right\rangle=\left\langle u, P(D)\left(\varphi_{l} \phi\right)\right\rangle=\left\langle u, \varphi_{l} P(D) \phi\right\rangle=\left\langle P(-D)\left(\varphi_{l} u\right), \phi\right\rangle
$$

for all $\phi \in \mathscr{D}\left(\Omega_{m_{l}}\right)$. Since the equation $P(-D) v=f$ has at most one solution with compact support (cf. [10, Theorem 7.3.2]), it follows that $u=\sum_{l=1}^{r} \varphi_{l} u$. Moreover, $\varphi_{l} u \in \mathscr{E}^{\prime}(\bar{\Omega})$ and $P(-D)\left(\varphi_{l} u\right)=\varphi_{l} f \in \mathscr{E}^{\prime}\left(\Omega_{m_{l}}\right)$, so that by hypothesis on $\Omega_{m_{l}}$ we have supp $\varphi_{l} u \subset \Omega_{m_{l}}$. Hence supp $u \subset \bigcup_{m} \Omega_{m}$.

THEOREM 4.4. Let $\Omega$ be an open subset of $\mathbb{R}^{N}$ and let $f_{m}: \Omega \rightarrow$ $\Omega_{m}, m \in \mathbb{N}$, be diffeomorphisms with $\Omega_{m} \subset \Omega$. Moreover, let $P$ be a nonconstant polynomial which is $f_{m}$-invariant for all $m \in \mathbb{N}$. If for every compact subset $K$ of $\Omega$ there are $m \in \mathbb{N}$ and $U \subset \Omega$ open with $K \subset U$ such that $f_{m}(U) \cup U$ is $P$-approximable in $\Omega$ and $f_{m}(U) \cap U=\emptyset$ then

$$
\mathcal{U}:=\left\{u \in \mathcal{N}_{P}(\Omega) ;\left(u \circ f_{m}\right)_{m \in \mathbb{N}} \text { is dense in } \mathcal{N}_{P}(\Omega)\right\}
$$

is a dense $G_{\delta}$-subset of $\mathcal{N}_{P}(\Omega)$.

Proof. Since $\mathcal{N}_{P}(\Omega)$ is a separable Fréchet space, by [8, Theorem 1] it suffices to show that for every pair of non-empty open subsets $V, W \subset$ $\mathcal{N}_{P}(\Omega)$ there is $m \in \mathbb{N}$ with $f_{m}^{*}(V) \cap W \neq \emptyset$.

In order to do so, let $\left(K_{n}\right)_{n \in \mathbb{N}}$ be a compact exhaustion of $\Omega$ and for $n \in \mathbb{N}$ choose $\varphi_{n} \in \mathscr{D}\left(\mathbb{R}^{N}\right)$ such that $\operatorname{supp} \varphi_{n} \subset K_{n+1}$ and $K_{n} \subset\left\{\varphi_{n}=1\right\}$. As mentioned in Section 2, the topology of $\mathcal{N}_{P}(\Omega)$ is generated by the increasing sequence of seminorms $q_{n}(u)=\max _{1 \leq j, k \leq n}\left\|\varphi_{k} u\right\|_{j}, n \in \mathbb{N}$.

Let $V, W$ be two non-empty open subsets of $\mathcal{N}_{P}(\Omega)$. Pick $v \in V$ and $w \in W$. Then there are $n \in \mathbb{N}$ and $\varepsilon>0$ such that

$$
\left\{u \in \mathcal{N}_{P}(\Omega) ; q_{n}(u-v)<\varepsilon\right\} \subset V, \quad\left\{u \in \mathcal{N}_{P}(\Omega) ; q_{n}(u-w)<\varepsilon\right\} \subset W .
$$

By hypothesis there are $m \in \mathbb{N}$ and $U \subset \Omega$ open with $K_{n+2} \subset U$ such that $f_{m}(U) \cap U=\emptyset$ and $f_{m}(U) \cup U$ is $P$-approximable. Since $f_{m}^{*}$ is continuous, there are $C \geq 1$ and $n^{\prime} \in \mathbb{N}$ such that

$$
\forall u \in \mathcal{N}_{P}(\Omega): \quad q_{n+1}\left(f_{m}^{*} u\right) \leq C q_{n^{\prime}}(u) .
$$

From the choice of $U$ it follows that $\varphi_{k} \in \mathscr{D}(U)$ as well as $\varphi_{k} \circ f_{m}^{-1} \in$ $\mathscr{D}\left(f_{m}(U)\right)$ for $1 \leq k \leq n$. We define $\tilde{u} \in \mathscr{D}^{\prime}\left(f_{m}(U) \cup U\right)$ via 


$$
\begin{aligned}
\forall \phi \in \mathscr{D}(U): & \langle\tilde{u}, \phi\rangle:=\langle v, \phi\rangle, \\
\forall \phi \in \mathscr{D}\left(f_{m}(U)\right): & \langle\tilde{u}, \phi\rangle:=\left\langle w \circ f_{m}^{-1}, \phi\right\rangle .
\end{aligned}
$$

Note that $\tilde{u}$ is well-defined since $f_{m}(U) \cap U=\emptyset$. Because $v \in \mathcal{N}_{P}(\Omega)$ and $w \circ f_{m}^{-1} \in \mathcal{N}_{P}\left(\Omega_{m}\right)$ it follows that $\tilde{u} \in \mathcal{N}_{P}\left(U \cup f_{m}(U)\right)$.

Because $\varphi_{k} \in \mathscr{D}(U)$ and $\varphi_{k} \circ f_{m}^{-1} \in \mathscr{D}\left(f_{m}(U)\right), 1 \leq k \leq n$, it follows that $q_{n}$ as well as $\max _{1 \leq k \leq n} q_{n^{\prime}}\left(\left(\varphi_{k} \circ f_{m}^{-1}\right) \cdot\right)$ are continuous seminorms on $\mathcal{N}_{P}\left(U \cup f_{m}(U)\right)$. By the $P$-approximability of $U \cup f_{m}(U)$ in $\Omega$ it follows that there is $u \in \mathcal{N}_{P}(\Omega)$ such that

$$
q_{n}(u-\tilde{u})<\varepsilon / C
$$

as well as

$$
\max _{1 \leq k \leq n} q_{n^{\prime}}\left(\left(\varphi_{k} \circ f_{m}^{-1}\right)(u-\tilde{u})\right)<\varepsilon / C .
$$

Since $\varphi_{k} \in \mathscr{D}(U), 1 \leq k \leq n$, we have $\varphi_{k} \tilde{u}=\varphi_{k} v$ so that

$$
q_{n}(u-v)=\max _{1 \leq k, j \leq n}\left\|\varphi_{k}(u-v)\right\|_{j}=\max _{1 \leq k, j \leq n}\left\|\varphi_{k}(u-\tilde{u})\right\|_{j}=q_{n}(u-\tilde{u})<\varepsilon
$$

i.e. $u \in V$. Moreover, because $\varphi_{k} \circ f_{m}^{-1} \in \mathscr{D}\left(f_{m}(U)\right), 1 \leq k \leq n$, we obtain $\left(\varphi_{k} \circ f_{m}^{-1}\right) \tilde{u}=\left(\varphi_{k} \circ f_{m}^{-1}\right)\left(w \circ f_{m}^{-1}\right), 1 \leq k \leq n$. From Corollary 3.5 and (2) applied to $w \circ f_{m}^{-1}-u$ it therefore follows that

$$
\begin{aligned}
q_{n}\left(w-u \circ f_{m}\right) & =q_{n}\left(f_{m}^{*}\left(w \circ f_{m}^{-1}-u\right)\right) \\
& \leq \max _{1 \leq k \leq n} q_{n+1}\left(f_{m}^{*}\left[\left(\varphi_{k} \circ f_{m}^{-1}\right)\left(w \circ f_{m}^{-1}-u\right)\right]\right) \\
& \leq C \max _{1 \leq k \leq n} q_{n^{\prime}}\left(\left(\varphi_{k} \circ f_{m}^{-1}\right)\left(w \circ f_{m}^{-1}-u\right)\right) \\
& =C \max _{1 \leq k \leq n} q_{n^{\prime}}\left(\left(\varphi_{k} \circ f_{m}^{-1}\right)(\tilde{u}-u)\right)<\varepsilon,
\end{aligned}
$$

i.e. $u \circ f_{m} \in W$ so that $f_{m}^{*}(V) \cap W \neq \emptyset$. Since $V, W$ were chosen arbitrarily, the conclusion follows from [8, Theorem 1].

REMARK 4.5. Let $P(D)$ be either the $\bar{\partial}$, Laplace, heat or wave operator. Moreover, consider the tempered weight functions $k_{j}(\xi)=(1+|\xi|)^{j}, j \in \mathbb{N}$, so that we are dealing with $\mathscr{E}_{P}(\Omega)$ as the kernel of $P(D)$. (Note, however, that this is no restriction for the $\bar{\partial}$, Laplace, or heat operator since these are hypoelliptic operators!) Let $f: \Omega \rightarrow \Omega_{1} \subset \Omega$ be a diffeomorphism such that $P$ is $f$-invariant, and in the case of the wave operator, assume that $f$ satisfies the additional mild conditions posed in Proposition 3.6(d).

Then by Proposition 3.6 a straightforward calculation shows that for all $\varphi \in \mathscr{E}(\Omega)$ one has

$$
P(D)(\varphi \circ f)=g((P(D) \varphi) \circ f)
$$


where

$$
g= \begin{cases}2 \bar{\partial} f_{1} & \text { if } P(D)=\bar{\partial} \\ \left|\nabla f_{1}\right|^{2} & \text { if } P(D) \text { is the Laplacian, } \\ \alpha^{2} \neq 0 & \text { if } P(D) \text { is the heat or wave operator }\end{cases}
$$

which has no zero in $\Omega$, because $f$ is a diffeomorphism. Hence, the following corollary covers the cases when $P(D)$ is the $\bar{\partial}$, Laplace, heat or wave operator.

In the case of the $\bar{\partial}$ operator, i.e. when dealing with holomorphic functions, the next result is due to Bernal and Montes (Theorem 1.2); cf. [2].

COROLlary 4.6. Let $\Omega$ be an open subset of $\mathbb{R}^{N}$ having convex components and let $P$ be a non-constant polynomial. Moreover, let $f_{m}: \Omega \rightarrow \Omega$, $m \in \mathbb{N}$, be diffeomorphisms of $\Omega$ such that for every $m \in \mathbb{N}$ there is $g_{m} \in \mathscr{E}(\Omega)$ having no zero in $\Omega$ such that $P(D)\left(f_{m}^{*} u\right)=g_{m} f_{m}^{*}(P(D) u)$ for every $u \in \mathscr{E}(\Omega)$ and $m \in \mathbb{N}$. Then $P$ is $f_{m}$-invariant for every $m \in \mathbb{N}$ and the following are equivalent.

(i) The set $\left\{u \in \mathscr{E}_{P}(\Omega) ;\left(u \circ f_{m}\right)_{m \in \mathbb{N}}\right.$ is dense in $\left.\mathscr{E}_{P}(\Omega)\right\}$ is a dense $G_{\delta}$-subset of $\mathscr{E}_{P}(\Omega)$.

(ii) There is $u \in \mathscr{E}_{P}(\Omega)$ such that $\left(u \circ f_{m}\right)_{m \in \mathbb{N}}$ is dense in $\mathscr{E}_{P}(\Omega)$.

(iii) For every compact subset $K$ of $\Omega$ there is $m \in \mathbb{N}$ such that $f_{m}(K)$ $\cap K=\emptyset$.

Proof. That $P$ is $f_{m}$-invariant for each $m$ follows immediately. Obviously, (i) implies (ii). In order to show that (iii) implies (i), observe that it follows immediately from the hypothesis on $\Omega$ that there is a compact exhaustion $\left(K_{n}\right)_{n \in \mathbb{N}}$ of $\Omega$ such that for every $n$ the components of $K_{n}$ are convex. By hypothesis, for every $n$ there is $m$ such that $f_{m}\left(K_{n}\right) \cap K_{n}=\emptyset$, i.e. the closures of $f_{m}\left(K_{n}^{\circ}\right)$ and $K_{n}^{\circ}$ are disjoint. The components of $K_{n}^{\circ}$ being convex, it follows that every $u \in \mathscr{E}^{\prime}(\bar{\Omega})$ with supp $P(-D) u \subset K_{n}^{\circ}$ satisfies $\operatorname{supp} u \subset K_{n}^{\circ}$. We also show that $\operatorname{supp} u \subset f_{m}\left(K_{n}^{\circ}\right)$ for every $u \in \mathscr{E}^{\prime}(\bar{\Omega})$ with supp $P(-D) u \subset f_{m}\left(K_{n}^{\circ}\right)$, so that $f_{m}\left(K_{n}^{\circ}\right) \cup K_{n}^{\circ}$ is $P$-approximable in $\Omega$ by Proposition 4.3 (ii). Since $\left(K_{n}\right)_{n \in \mathbb{N}}$ is a compact exhaustion of $\Omega$, this will show that the hypothesis of Theorem 4.4 is satisfied, giving (i). In order to simplify notation, we simply write $f$ instead of $f_{m}$ from now on.

So, let $u \in \mathscr{E}^{\prime}(\bar{\Omega})$ with $\operatorname{supp} P(-D) u \subset f\left(K_{n}^{\circ}\right)$. As $\Omega$ has convex components, it follows that $\operatorname{supp} u \subset \Omega$, so we can apply $f^{*}$ to $u$. By hypothesis we have

$$
P(D)(\varphi \circ f)=g((P(D) \varphi) \circ f)
$$

for all $\varphi \in \mathscr{E}(\Omega)$, where $g$ has no zero in $\Omega$. In particular $P(D) \varphi=$ $g\left(\left(P(D)\left(\varphi \circ f^{-1}\right)\right) \circ f\right)$. For $v \in \mathscr{D}^{\prime}(\Omega)$ and $\varphi \in \mathscr{D}(\Omega)$ we therefore have 


$$
\begin{aligned}
\langle P(-D) & (v \circ f), \varphi\rangle=\left\langle v,\left|\operatorname{det} J f^{-1}\right|(P(D) \varphi) \circ f^{-1}\right\rangle \\
& =\left\langle v,\left|\operatorname{det} J f^{-1}\right|\left(g \circ f^{-1}\right) P(D)\left(\varphi \circ f^{-1}\right)\right\rangle \\
& =\left\langle P(-D)\left(\left|\operatorname{det} J f^{-1}\right|\left(g \circ f^{-1}\right) v\right), \varphi \circ f^{-1}\right\rangle \\
& =\left\langle P(-D)\left(\left|\operatorname{det} J f^{-1}\right|\left(g \circ f^{-1}\right) v\right),\left|\operatorname{det}(J f) \circ f^{-1}\right|\left|\operatorname{det} J f^{-1}\right| \varphi \circ f^{-1}\right\rangle \\
& =\left\langle f^{*}\left(P(-D)\left(\left|\operatorname{det} J f^{-1}\right|\left(g \circ f^{-1}\right) v\right),|\operatorname{det} J f| \varphi\right\rangle\right. \\
& =\left\langle|\operatorname{det} J f| f^{*}\left(P(-D)\left(\left|\operatorname{det} J f^{-1}\right|\left(g \circ f^{-1}\right) v\right), \varphi\right\rangle,\right.
\end{aligned}
$$

i.e. $P(-D)\left(f^{*} v\right)=|\operatorname{det} J f| f^{*}\left(P(-D)\left(\left|\operatorname{det} J f^{-1}\right|\left(g \circ f^{-1}\right) v\right)\right)$ for all $v \in$ $\mathscr{D}^{\prime}(\Omega)$. Therefore,

$$
P(-D) u=\left(f^{-1}\right)^{*}\left[\frac{1}{|\operatorname{det} J f|} P(-D)\left[f^{*}\left(\frac{1}{\left|\operatorname{det} J f^{-1}\right|\left(g \circ f^{-1}\right)} u\right)\right],\right.
$$

which shows

$$
\begin{aligned}
\operatorname{supp}\left(f^{-1}\right)^{*}\left[\frac{1}{|\operatorname{det} J f|} P(-D)\left[f^{*}\left(\frac{1}{\left|\operatorname{det} J f^{-1}\right|\left(g \circ f^{-1}\right)} u\right)\right]\right] \\
=\operatorname{supp} P(-D) u \subset f\left(K_{n}^{\circ}\right) .
\end{aligned}
$$

From Proposition 3.1 we get

$$
\operatorname{supp} \frac{1}{|\operatorname{det} J f|} P(-D)\left[f^{*}\left(\frac{1}{\left|\operatorname{det} J f^{-1}\right|\left(g \circ f^{-1}\right)} u\right)\right] \subset K_{n}^{\circ} \text {. }
$$

Since $1 /|\operatorname{det} J f| \neq 0$ we get

$$
\operatorname{supp} P(-D)\left[f^{*}\left(\frac{1}{\left|\operatorname{det} J f^{-1}\right|\left(g \circ f^{-1}\right)} u\right)\right] \subset K_{n}^{\circ},
$$

so that by the convexity of the components of $K_{n}^{\circ}$,

$$
\operatorname{supp} f^{*}\left(\frac{1}{\left|\operatorname{det} J f^{-1}\right|\left(g \circ f^{-1}\right)} u\right) \subset K_{n}^{\circ} .
$$

Using Proposition 3.1 once more gives

$$
\operatorname{supp} \frac{1}{\left|\operatorname{det} J f^{-1}\right|\left(g \circ f^{-1}\right)} u \subset f\left(K_{n}^{\circ}\right),
$$

hence supp $u \subset f\left(K_{n}^{\circ}\right)$, finally giving (i) of the corollary.

Observe that this last conclusion is the only one where we used that $f(\Omega)=\Omega$, for if this was not the case we would only obtain

$$
f(\Omega) \cap \operatorname{supp} \frac{1}{\left|\operatorname{det} J f^{-1}\right|\left(g \circ f^{-1}\right)} u \subset f\left(K_{n}^{\circ}\right)
$$

from Proposition 3.5

Finally, that (ii) implies (iii) is shown exactly as in [2, Theorem 3.5]. Assume there is a compact subset $K$ of $\Omega$ such that $f_{m}(K) \cap K \neq \emptyset$ for all $m \in \mathbb{N}$. So, there are $x_{m} \in K$ with $f_{m}\left(x_{m}\right) \in K$ for every $m$. Since in 
every case under consideration, $P$ is a non-constant polynomial, the function $v(x)=1+\max _{y \in K}|u(y)|$ belongs to $\mathscr{E}_{P}(\Omega)$. For every $m$ we have

$\max _{x \in K}\left|v(x)-u\left(f_{m}(x)\right)\right| \geq\left|v\left(x_{m}\right)-u\left(f_{m}\left(x_{m}\right)\right)\right| \geq\left|v\left(x_{m}\right)\right|-\left|u\left(f_{m}\left(x_{m}\right)\right)\right| \geq 1$, contradicting the denseness of $\left(u \circ f_{m}\right)_{m \in \mathbb{N}}$ in $\mathscr{E}_{P}(\Omega)$.

ExAmple 4.7. Let $\Omega=B_{1}(0) \times \mathbb{R} \subset \mathbb{R}^{N+1}$ with $B_{1}(0)=\left\{x^{\prime} \in \mathbb{R}^{N} ;\left|x^{\prime}\right|<1\right\}$. Moreover, let $A_{m} \in \mathbb{R}^{N \times N}$ be an orthogonal matrix and $b_{m} \in \operatorname{span}\left\{e_{N+1}\right\}$, where $e_{N+1}$ is the $(N+1)$ th unit vector in $\mathbb{R}^{N+1}$. Clearly,

$$
f_{m}: \Omega \rightarrow \Omega, \quad\left(x^{\prime}, x_{N+1}\right) \mapsto\left(A_{m} x^{\prime}, x_{N+1}\right)+b_{m},
$$

is a well-defined diffeomorphism.

By Proposition 3.6, both polynomials $P_{H}(\xi)=|\xi|^{2}+i \xi_{N+1}$ and $P_{W}(\xi)=$ $|\xi|^{2}-\xi_{N+1}^{2}$ are $f_{m}$-invariant for every $m \in \mathbb{N}$. $P_{H}(D)$ gives the heat operator, whereas $P_{W}(D)$ gives the wave operator.

It follows from Corollary 4.6 and Remark 4.5 that there is an $\left(f_{m}\right)_{m \in \mathbb{N}^{-}}$ universal zero solution of the heat operator, respectively the wave operator, if and only if limsup $\sup _{m \rightarrow \infty}\left|b_{m}\right|=\infty$. While sufficiency of this condition is obvious, to show necessity assume that $\left(\left|b_{m}\right|\right)_{m \in \mathbb{N}}$ is bounded by a constant $C$. Let $K=\left\{x^{\prime} \in \mathbb{R}^{N} ;\left|x^{\prime}\right| \leq 1 / 2\right\} \times[-C, C]$. Then $0 \in K \cap f_{m}(K)$ for all $m \in \mathbb{N}$, so that necessity follows from Corollary 4.6 too.

5. Dense subspaces of universal zero solutions. Under a slight modification of the hypothesis of Theorem 4.4 one can even prove the following stronger result.

THEOREM 5.1. Let $\Omega$ be an open subset of $\mathbb{R}^{N}$, and $f_{m}: \Omega \rightarrow \Omega_{m}$, $m \in \mathbb{N}$, diffeomorphisms with $\Omega_{m} \subset \Omega$. Moreover, let $P$ be a non-constant polynomial which is $f_{m}$-invariant for all $m \in \mathbb{N}$. If for every compact subset $K$ of $\Omega$ there are $m \in \mathbb{N}$ and $U \subset \Omega$ open and bounded with $K \subset U \subset \bar{U} \subset \Omega$ such that $f_{m}(U) \cap U=\emptyset$ and $f_{m}(U) \cup U$ is $P$-approximable in $\Omega$ then there is a dense subspace $\mathcal{L} \subset \mathcal{N}_{P}(\Omega)$ with

$\mathcal{L} \backslash\{0\} \subset \mathcal{U}:=\left\{u \in \mathcal{N}_{P}(\Omega) ;\left(u \circ f_{m}\right)_{m \in \mathbb{N}}\right.$ is dense in $\left.\mathcal{N}_{P}(\Omega)\right\}$.

Proof. Let $\left(K_{n}\right)_{n \in \mathbb{N}}$ be a compact exhaustion of $\Omega$. By the hypothesis we construct inductively an increasing sequence of open, bounded subsets $\left(U_{n}\right)_{n \in \mathbb{N}}$ of $\Omega$ with $K_{n} \subset U_{n} \subset \overline{U_{n}} \subset \Omega$ and a strictly increasing sequence $\left(m_{n}\right)_{n \in \mathbb{N}}$ of positive integers such that

(i) $\forall n \in \mathbb{N}: f_{m_{n}}\left(U_{n}\right) \cup U_{n}$ is $P$-approximable in $\Omega$,

(ii) $\forall n \in \mathbb{N}: f_{m_{n}}\left(U_{n}\right) \cap U_{n}=\emptyset$.

For $n=1$ by hypothesis there are $m_{1} \in \mathbb{N}$ and $U_{1} \subset \Omega$ open and bounded such that $K_{1} \subset U_{1} \subset \overline{U_{1}} \subset \Omega, f_{m_{1}}\left(U_{1}\right) \cap U_{1}=\emptyset$, and $f_{m_{1}}\left(U_{1}\right) \cup U_{1}$ is $P$-approximable in $\Omega$. 
If $U_{1}, \ldots, U_{n}, m_{1}, \ldots, m_{n}$ have been constructed we apply the hypothesis to the compact set

$$
\overline{U_{n}} \cup \overline{f_{1}\left(U_{n}\right)} \cup \cdots \cup \overline{f_{m_{n}}\left(U_{n}\right)} \cup K_{n+1}
$$

to obtain some $m_{n+1} \in \mathbb{N}$ and $U_{n+1} \subset \Omega$ open and bounded such that

$$
\overline{U_{n}} \cup \overline{f_{1}\left(U_{n}\right)} \cup \cdots \cup \overline{f_{m_{n}}\left(U_{n}\right)} \cup K_{n+1} \subset U_{n+1} \subset \overline{U_{n+1}} \subset \Omega
$$

with $f_{m_{n+1}}\left(U_{n+1}\right) \cap U_{n+1}=\emptyset$ and $f_{m_{n+1}}\left(U_{n+1}\right) \cup U_{n+1} P$-approximable in $\Omega$. In particular, from $f_{m_{n+1}}\left(U_{n+1}\right) \cap U_{n+1}=\emptyset$ it follows that for all $1 \leq j \leq m_{n}$ we have $f_{m_{n+1}}\left(U_{n}\right) \cap f_{j}\left(U_{n}\right)=\emptyset$, hence $m_{n+1}>m_{n}$.

We will now show that for the subsequence $\left(f_{m_{n}}\right)_{n \in \mathbb{N}}$ there is a dense linear subspace $\mathcal{L}$ of $\mathcal{N}_{P}(\Omega)$ such that $\left(u \circ f_{m_{n}}\right)_{n \in \mathbb{N}}$ is dense in $\mathcal{N}_{P}(\Omega)$ for every $u \in \mathcal{L} \backslash\{0\}$, proving the theorem. Since we will be dealing with subsequences of $\left(f_{m_{n}}\right)_{n \in \mathbb{N}}$ we simply write $\left(f_{m}\right)_{m \in \mathbb{N}}$ instead of $\left(f_{m_{n}}\right)_{n \in \mathbb{N}}$ to simplify notation.

Let $\left(f_{m_{n}}\right)_{n \in \mathbb{N}}$ be an arbitrary subsequence of $\left(f_{m}\right)_{m \in \mathbb{N}}$. For a given compact subset $K \subset \Omega$ there is $n \in \mathbb{N}$ such that $K \subset K_{m_{n}} \subset U_{m_{n}}$ and $f_{m_{n}}\left(U_{m_{n}}\right) \cap U_{m_{n}}=\emptyset$ and $f_{m_{n}}\left(U_{m_{n}}\right) \cup U_{m_{n}}$ is $P$-approximable in $\Omega$. Therefore, $\left\{u \in \mathcal{N}_{P}(\Omega) ;\left(u \circ f_{m_{n}}\right)_{n \in \mathbb{N}}\right.$ is dense in $\left.\mathcal{N}_{P}(\Omega)\right\}$ is a dense subset of $\mathcal{N}_{P}(\Omega)$ by Theorem 4.4. Since $\left(f_{m_{n}}\right)_{n \in \mathbb{N}}$ is an arbitrary subsequence of $\left(f_{m}\right)_{m \in \mathbb{N}}$ it follows from [1, Theorem 2] that there is a dense linear subspace $\mathcal{L}$ of $\mathcal{N}_{P}(\Omega)$ such that $\left(u \circ f_{m}\right)_{m \in \mathbb{N}}$ is dense in $\mathcal{N}_{P}(\Omega)$ for every $u \in \mathcal{L} \backslash\{0\}$.

Referring to Theorem 5.1 rather than Theorem 4.4 , the proof of Corollary 4.6 gives the result below. By Remark 4.5 , in the case of the $\bar{\partial}$, Laplace, heat and wave operator its hypothesis on the diffeomorphisms $f_{m}$ is automatically satisfied if the corresponding polynomial is $f_{m}$-invariant.

Corollary 5.2. Let $\Omega$ be an open subset of $\mathbb{R}^{N}$ having convex components and let $P$ be a non-constant polynomial. Moreover, let $f_{m}: \Omega \rightarrow \Omega$, $m \in \mathbb{N}$, be diffeomorphisms of $\Omega$ such that for every $m \in \mathbb{N}$ there is $g_{m} \in \mathscr{E}(\Omega)$ having no zero in $\Omega$ such that $P(D)\left(f_{m}^{*} u\right)=g_{m} f_{m}^{*}(P(D) u)$ for every $u \in \mathscr{E}(\Omega)$. Then $P$ is $f_{m}$-invariant for every $m \in \mathbb{N}$ and the following are equivalent.

(i) There is a dense subspace $\mathcal{L} \subset \mathscr{E}_{P}(\Omega)$ with

$$
\mathcal{L} \backslash\{0\} \subset\left\{u \in \mathscr{E}_{P}(\Omega) ;\left(u \circ f_{m}\right)_{m \in \mathbb{N}} \text { is dense in } \mathscr{E}_{P}(\Omega)\right\} .
$$

(ii) The set $\left\{u \in \mathscr{E}_{P}(\Omega) ;\left(u \circ f_{m}\right)_{m \in \mathbb{N}}\right.$ is dense in $\left.\mathscr{E}_{P}(\Omega)\right\}$ is a dense $G_{\delta}$-subset of $\mathscr{E}_{P}(\Omega)$.

(iii) There is $u \in \mathscr{E}_{P}(\Omega)$ such that $\left(u \circ f_{m}\right)_{m \in \mathbb{N}}$ is dense in $\mathscr{E}_{P}(\Omega)$.

(iv) For every compact subset $K$ of $\Omega$ there is $m \in \mathbb{N}$ such that $f_{m}(K)$ $\cap K=\emptyset$. 
Acknowledgements. We thank the referee, especially for pointing out [1, Theorem 2] which simplified the proof of Theorem 5.1 considerably.

\section{References}

[1] L. Bernal-González, Densely hereditarily hypercyclic sequences and large hypercyclic manifolds, Proc. Amer. Math. Soc. 127 (1999), 3279-3285.

[2] L. Bernal-González and A. Montes-Rodríguez, Universal functions for composition operators, Complex Variables 27 (1995), 47-56.

[3] G. D. Birkhoff, Démonstration d'un théorème élémentaire sur les fonctions entières, C. R. Acad. Sci. Paris 189 (1929), 473-475.

[4] M. C. Calderón-Moreno and J. Müller, Universal holomorphic and harmonic functions with additional properties, Acta Math. Hungar. 105 (2004), 1-15.

[5] S. M. Duyos-Ruiz, Universal functions of the structure of the space of entire functions, Soviet Math. Dokl. 30 (1984), 713-716.

[6] O. P. Dzagnidze, The universal harmonic function in the space $E_{n}$, Sakharth. SSR Mecn. Akad. Moambe 55 (1969), 41-44.

[7] P. M. Gauthier and M. R. Pouryayevali, Universal (pluri)subharmonic functions, Analysis 27 (2007), 273-284.

[8] K. G. Grosse-Erdmann, Universal families and hypercyclic operators, Bull. Amer. Math. Soc. 36 (1999), 345-381.

[9] K. G. Grosse-Erdmann and R. Mortini, Universal functions for composition operators with non-automorphic symbol, J. Anal. Math. 107 (2009), 355-376.

[10] L. Hörmander, The Analysis of Linear Partial Differential Operators I and II, Springer, Berlin, 1983.

[11] W. Luh, Universalfunktionen in einfach zusammenhängenden Gebieten, Aequationes Math. 19 (1979), 183-193.

[12] R. Meise and D. Vogt, Introduction to Functional Analysis, Clarendon Press, Oxford, 1997.

[13] W. Rudin, Functional Analysis, McGraw-Hill, New York, 1973.

[14] W. P. Seidel and J. L. Walsh, On approximation by Euclidean and non-Euclidean translates of an analytic function, Bull. Amer. Math. Soc. 47 (1941), 916-920.

[15] F. Treves, Topological Vector Spaces, Distributions and Kernels, Academic Press, New York, 1967.

[16] P. Zappa, On universal holomorphic functions, Boll. Un. Mat. Ital. A (7) (1989), 345-352.

Thomas Kalmes

FB IV - Mathematik

Universität Trier

54286 Trier, Germany

E-mail: kalmesth@uni-trier.de
Markus Niess Katholische Universität Eichstätt-Ingolstadt Mathematisch-Geographische Fakultät D-85071 Eichstätt, Germany E-mail: markus.niess@ku-eichstaett.de 